\title{
Sentando las bases para la indización: cambios en las instrucciones para los autores de Acta Médica Peruana
}

\section{Setting the bases for being included in Medline and Scopus: changes in instructions for authors in Acta Medica Peruana}

Recibido: 02/05/2016

Aprobado: 04/05/2016

Citar como:

Zolezzi A, Mayta-Tristán P. Sentando las bases para la indización: cambios en las instrucciones para los autores de Acta Médica Peruana. Acta Med Peru. 2016;33(1):7-8

\author{
Alberto Zolezzi' ${ }^{1}$ Percy Mayta-Tristán ${ }^{1,2}$ \\ 1 Acta Médica Peruana, Colegio Médico del Perú. Lima, Perú. \\ 2 Escuela de Medicina, Universidad Peruana de Ciencias Aplicadas. Lima, Perú.
}

En 1972 el Colegio Médico del Perú (CMP) publica el primer número de Acta Médica Peruana (AMP), su revista oficial, que buscaba inicialmente difundir los conocimientos y experiencias clínicas de los médicos peruanos. En estos 44 años de existencia ha habido periodos de silencio editorial, en las décadas del 70 (1976-77), la década del 80 (1988-1990) y la década del 90 (1994-1998); sin embargo, desde 1999 la revista se publicó en forma ininterrumpida, aunque hubo problemas en el año 2015 que fueron resueltos con oportunidad [1]. Para que una revista sea exitosa, debe tener el apoyo institucional pero con independencia editorial, un equipo que conozca de los proceso editoriales que incluye la selección de revisores idóneos, continuidad, responsabilidad, sentido ético y compromiso con la calidad. En ese sentido, la nueva gestión del CMP ha encomendado al comité editorial de AMP el reto de alcanzar la cantidad de artículos con la calidad exigida para postularse a MEDLINE y Scopus. Por lo que uno de los primeros pasos es adaptar las instrucciones para los autores de AMP [2] a requerimientos de excelencia. En esta editorial vamos a detallar los cambios más importantes, que podemos resumirlos en (a) requerimientos éticos, (b) autoría, (c) tipos de artículos, (d) adherencia a consensos internacionales de presentación de artículos, (e) adopción del Open Journal System como sistema de envío, revisión y publicación de artículos.

Toda investigación realizada a partir del año 2016 que sea presentada a AMP y que sea realizada en humanos o usen material biológico o datos susceptibles de identificación como muestras o registros médicos debe contar con la aprobación de un Comité de Ética en Investigación excepto 1) Estudios con bases de datos secundarios de acceso público, incluyendo revisiones sistemáticas, meta-análisis y estudios bibliométricos; 2 ) Intervenciones de vigilancia en salud pública; 3) Investigación de brotes o emergencias sanitarias; 4) Evaluaciones de programas de salud pública; 5) Evaluaciones educativas programadas dentro del currículo académico [3]. Con estudios realizados antes del 2016 donde no se cuente con una aprobación por un Comité de Ética, el comité editorial de AMP evaluará cada caso.

Las instrucciones previas de AMP mencionaban que solo podían ser autores corresponsales los médicos afiliados al CMP, esto limitaba la apertura de contenidos a colegas extranjeros así como investigadores no médicos que tienen también mucho por aportar a la revista. Además, esto se contraponía con las recomendaciones de los criterios de autoría del International Committee of Medical Journal Editor [4], que establece cuatro criterios a cumplir para considerar a una persona como autor de un artículo, por lo que el grado académico de las personas o la profesión no son determinantes para la autoría, sino solo la contribución al desarrollo del mismo.

Entre los tipos de artículo, se diferencia dentro de las cartas al editor a aquellas que son resultados de investigación, denominadas cartas científicas y tienen diferente extensión que las cartas al editor en general, entre las cuales tienen como prioridad aquellas que responden a un artículo publicado en el último número de AMP, las que aportan a la política editorial de la revista o plantean temas de interés para la práctica 
clínica: educación médica, política sanitaria del país o vinculados al ejercicio profesional de la medicina, o denuncias relacionadas con faltas éticas en publicación de algún artículo publicado.

Adicionalmente se crea un nuevo tipo de artículo, protocolos de investigación, solo será para aquellos estudios probabilísticos multietápicos a nivel regional o país, estudios multicéntricos, ensayos clínicos o grandes cohortes que tengan la posibilidad de generar varios artículos posteriores del análisis de su data, por lo que se requiere disponer las características principales de las mediciones y proceso de selección muestral que no se pueden describir en cada análisis secundario. Creemos que esta nueva sección ayudará a conocer las bases de datos disponibles, así como proyectos de interés que se vienen desarrollando en el país.

En cuanto a las recomendaciones para los artículos de investigación se recomienda a todos los autores seguir las recomendaciones específicas para cada tipo de diseño como STROBE para observacionales [5], STARD para pruebas diagnósticas [6], CONSORT para ensayos clínicos [7], PRISMA para revisiones sistemáticas [8], SRQR para estudios cualitativos [9], CHEERS para evaluaciones económicas [10] y CARE para los reportes de caso [11].

Hemos implementado ya el sistema de Open Journal Systems para Acta Médica Peruana, donde gradualmente iremos incorporando toda la colección previa de la revista, pero sobre todo será el único medio por donde se recibirán las nuevas propuestas de artículos para su revisión. La dirección electrónica de la revista es: www.amp.cmp.org.pe.

Finalmente, queremos reafirmar nuestro compromiso en llevar a Acta Médica Peruana a su pronta indización a Medline y Scopus, y si bien para los médicos ha sido siempre un plus poder publicar en la revista del Colegio Médico del Perú, queremos que ese plus signifique además la certeza que se ha publicado porque ha pasado los filtros de calidad (revisión por el comité editorial y por pares expertos en el tema), y que su artículo esté disponible en las bases de datos donde cualquier médico e investigador del mundo las busca: Medline y Scopus.

\section{REFERENCIAS BIBLIOGRÁFICAS}

1. Palacios-Celi M. Acta Médica Peruana: retomando el camino hacia la indización. Acta Med Peru. 2016;X(1):1-2.

2. Acta Médica Peruana. Instrucciones para los autores. Acta Med Peru. 2016;33(1):87-91

3. Arroyo-Hernandez $\mathrm{H}$, Cabezas C. Política editorial sobre la ética de investigaciones en humanos. Rev Peru Med Exp Salud Publica. 2015;32(1):9-10.

4. International Committee of Medical Journal Editor [Internet]. Defining the role of authors and contributors. Vancouver: ICMJE; 2015 [citado el 16 de febrero de 2016]. Disponible en: http://www.icmje.org/recommendations/browse/roles-andresponsibilities/defining-the-role-of-authors-and-contributors. html

5. Von Elm E, Altman DG, Egger M, Pocock SJ, Gøtzsche PC, Vandenbroucke JP, et al. The Strengthening the Reporting of Observational Studies in Epidemiology (STROBE) statement: guidelines for reporting observational studies. PLoS Med. 2007;4(10):e296.

6. Bossuyt PM, Reitsma JB, Bruns DE, Gatsonis CA, Glasziou PP, Irwig $L$, et al. STARD 2015: an updated list of essential items for reporting diagnostic accuracy studies. BMJ. 2015;351:h5527.

7. Schulz KF, Altman DG, Moher D; CONSORT Group. CONSORT 2010 Statement: updated guidelines for reporting parallel group randomised trials. Trials. 2010;11:32.

8. Moher D, Liberati A, Tetzlaff J, Altman DG; PRISMA Group. Preferred reporting items for systematic reviews and meta-analyses: the PRISMA statement. J Clin Epidemiol. 2009;62(10):1006-12.

9. O'Brien BC, Harris IB, Beckman TJ, Reed DA, Cook DA. Standards for reporting qualitative research: a synthesis of recommendations. Acad Med. 2014;89(9):1245-51.

10. Husereau D, Drummond M, Petrou S, Carswell C, Moher D, Greenberg D, et al. Consolidated Health Economic Evaluation Reporting Standards (CHEERS) statement. Eur J Health Econ. 2013;14(3):367-72.

11. Gagnier JJ, Kienle G, Altman DG, Moher D, Sox H, Riley D, et al. The CARE guidelines: consensus-based clinical case reporting guideline development. J Med Case Rep. 2013;7:223.

\title{
Ahora puede enviar sus artículos para Acta Médica Peruana en nuestro Open Journal System:
}

\author{
www.amp.cmp.org.pe
}

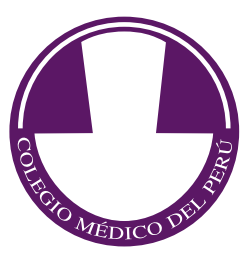

